\title{
Standards of teeth preparations for anterior resin bonded all-ceramic crowns in private dental practice in Jordan
}

\author{
Ziad Nawaf AL-DWAIRI ${ }^{1}$, Ahmad Saleh AL-HIYASAT ${ }^{2}$, Haitham ABOUD ${ }^{3}$ \\ 1- BDS, PhD (UK), Chairman, Prosthodontics Department, Jordan University of Science and Technology, Irbid, Jordan. \\ 2- BDS, MScD, PhD, Department of Conservative Dentistry, Faculty of Dentistry, Jordan University of Science and Technology, Irbid, Jordan. \\ 3- BDS, MSc, Graduate student, Faculty of Dentistry, Jordan University of Science and Technology, Irbid, Jordan.
}

Corresponding address: Ziad Nawaf AL-Dwairi - Associate Professor of Prosthodontics - Department of Prosthodontics/ Faculty of Dentistry - Jordan University of Science and Technology - P.O. Box: 303022110 - Irbid - Jordan - e-mail: ziadd@just.edu.jo

Received: June 18, 2009 - Modification: May 25, 2010 - Accepted: October 26, 2010

\section{ABSTRACT}

\begin{abstract}
$\mathrm{O}$ bjectives: To investigate if general dental practitioners (GDPs) in private practice in Jordan follow universal guidelines for preparation of anterior teeth for resin bonded all-ceramic crowns (RBCs). Material and Methods: A sample $(n=100)$ of laboratory models containing 208 tooth preparations for IPS Empress and In Ceram, featuring work from different GDPs, was obtained from 8 commercial dental laboratories. Aspects of preparations were quantified and compared with accepted criteria defined following a review of the literature and recommendations of the manufactures' guidelines. Results: Subgingival margins on the buccal aspect were noticed in $36 \%$ of the preparations, $54 \%$ demonstrated overpreparation with a tendency to overprepare the teeth on the mesiodistal plane more than buccolingual plane. Twenty percent of samples presented a shoulder finish line while a chamfer margin design was noticed in $39 \%$. Twenty-nine percent and $12 \%$ of samples had either a feathered or no clear margin design respectively. Incisal underpreparation was observed in $18 \%$ of dies of each type. Only $17 \%$ of all preparations were found to follow the recommended anatomical labial preparations while $29 \%$ of the RBC preparations were found to have the recommended axial convergence angle. In total, $43 \%$ of preparations were found to have the recommended depth of the finish line. Conclusions: It was found that relevant guidelines for RBC preparations were not being fully adhered to in private practice in Jordan.
\end{abstract}

Key words: Porcelain. Anterior teeth. In-ceram. Jordan. Private practice.

\section{INTRODUCTION}

As all-ceramic crowns have become one of the best aesthetic restorative materials, the need for good practice and skills to perform such restorations within patient expectations and the recommended guidelines for tooth preparations becomes mandatory ${ }^{33}$.

All-ceramic resin bonded crowns appear to have a number of advantages compared with conventional metal-ceramic crowns. First, their better aesthetic properties may be due to the fact that the composite resin luting material is more translucent than conventional cements used with porcelain fused to metal crowns, which improves the transmission of light through the restored unit, and because of a good peripheral blend at the gingival margin without a black-line margin due to the metal substructure ${ }^{5}$. Second, the gingival response may be better, given that the periodontal response to porcelain known to be relatively excellent ${ }^{12}$. Furthermore, given the insoluble nature of the resin luting material, the periodontal response associated with dentinbonded all-ceramic crowns may be superior to that associated with conventional crowns in which the luting agent at the margins may dissolve, resulting in possible plaque accumulation as well as a risk of caries lesion formation. Third, laboratory studies have shown that the fracture resistance of dentinbonded all-ceramic crowns was good, even though 
minimal preparations were used 9 .

When comparing porcelain-fused-to-metal to all-ceramic crowns, patient selection and technique sensitivity may be more critical with all-ceramic than with metal-ceramic restoration ${ }^{2,8}$. Furthermore, the coping design and luting system may be critical to maximize long-term success ${ }^{14}$.

Tooth preparation is one of the important aspects of restorative dentistry because it establishes the foundation for whatever restoration is being placed. Unfortunately, training in dental schools relative to tooth preparation is too often oriented to the dimensions of rotary instruments rather than tooth morphology. Understanding of tooth morphology is essential for developing preparations that will permit the restorations placed upon them to be functionally durable, provide optimal esthetics, and be biologically compatible with the periodontal tissues ${ }^{28}$.

In general, preparation principles applied in allceramic systems are comparable. The margin design should be either modified shoulder and rounded internal angles or chamfer. The prepared tooth should have a taper of $6^{\circ}$ to $10^{\circ}$. All contours should be smoothened and rounded off to reduce the risk of stress concentration areas in the ceramic, facilitate impression making, die pouring, fabrication of the restoration and cementation. Undercuts should be blocked out using a glass ionomer material or dentin-bonded composite ${ }^{18}$. The occlusal clearance should be a minimum of 1 millimeter in centric relation and lateral excursions. The removal of the tooth structure correlates to the manufacturer's guidelines. Overall, the preparation should be as conservative as possible with retention of some enamel if possible but in case of sever discoloration, minimum reduction maybe insufficient to provide adequate porcelain depth to cover the discoloration.

The marginal preparations should produce an optimal peripheral seal from restoration to tooth and should be supragingival as possible, because achieving isolation for the bonding and luting procedure may be difficult in subgingival areas. Furthermore, margins ideally should be on enamel, where marginal microleakage may be reduced compared with dentinal margins. Therefore, the margins should be well adapted, not deformed during function and be accessible to the dentist for finishing and for the patient for cleaning.

Variations in tooth preparation for RBCs are well seen among general dental practitioners (GDPs) around the world. Sutton and McCord ${ }^{32}$ (2001) showed that $29 \%$ of the preparations on the buccal aspect had subgingival margins and the majority of the margins ( $84 \%$ buccally and $79 \%$ lingually) of the dies examined exhibited appropriate shoulder or chamfer finishes.

Begazo, et al. ${ }^{3}$ (2004) found that the average values of all preparation parameters of all-ceramic crowns investigated were within the borders as defined in the preparation guidelines of the manufacturer. However, on an individual tooth level, nearly all preparations showed to have one or more locations with imperfections. Although several studies have discussed the importance of proper tooth preparation techniques that provide optimal integrity and increase longevity of the existing restoration, there were few studies that discussed the dental practitioner's clinical performance following these guidelines in their private practice, therefore, this study aims to find out the variations in preparing anterior teeth for all-ceramic crowns to show if the preparation techniques follow the recommended guidelines.

This study is based on analysis of samples of dies, which were prepared to receive resinbonded all-ceramic crowns (RBCs) for anterior teeth obtained from dental laboratories in Jordan. The results deducted from this research will show the most common clinical errors in preparations of anterior teeth for all-ceramic crowns between general dental practitioners in Jordan and will focus on the most accepted recommendations needed when preparing anterior teeth for all-ceramic crowns, which will ultimately lead to increased life time expectancy of the prosthesis, enhanced clinical performance, increased procedural efficiency, and elevated prosthesis quality.

\section{MATERIAL AND METHODS}

One hundred $(n=100)$ of laboratory models featuring tooth preparations for RBCs for anterior teeth from different general dental practitioners were chosen from private dental laboratories in 2 major cities in Jordan (Amman and Irbid). All dies have been examined visually and have been found to be sound without defects or cracks.

All samples included master casts supplied directly from dental laboratories containing sound anterior teeth of maxillary or mandibular jaw from canine to canine (investigation area) before die preparation. The ceramic crown systems available in dental laboratories in Jordan investigated included: In-Ceram (VITA Zahnfabrik H, Rauter GmbH \& Co., Bad Säckingen, Germany) and IPS Empress (Ivoclar Vivadent Inc., Schaan, Liechtenstein).

The master casts were first used for measurement of the tooth margin positions in relation to the gingival margin positions on the buccal and lingual aspects before die trimming and then all master cast were trimmed to carry on the rest of the measurements as mentioned below.

A specially designed wax cylinder $(23 \mathrm{~mm}$ length and $20 \mathrm{~mm}$ width) was fabricated to hold the prepared die in position. The preparation criteria 
investigated in this study included:

1. The positions of tooth preparation margin in relation to the gingival margin on the buccal and lingual aspects. This was measured before die trimming using Williams periodontal probe (Ash) ${ }^{21}$ according to the following criteria:

1) $>2 \mathrm{~mm}$ supragingival margin

2) $\leq 2 \mathrm{~mm}$ supragingival margin

3) Subgingival margin

4) Level with gingival margin

5) No clear margin

2. The total amount of tooth reduction in the buccolingual and mesiodistal planes. The measurements were carried out using digital vernier caliper and calculated by deducting the width of the prepared teeth from the unprepared contralateral tooth width in the two planes ${ }^{17}$. It was assessed according to the following criteria:

1) $>3 \mathrm{~mm}$ (Overpreparation)

2) $\leq 3 \mathrm{~mm}>2 \mathrm{~mm}$ (Recommended)

3) $\leq 2 \mathrm{~mm}>1 \mathrm{~mm}$ (Recommended)

4) $\leq 1 \mathrm{~mm}$ and $0 \mathrm{~mm}$ (Underpreparation)

3. The amount of incisal reduction. This was measured using the digital caliper by comparison to contralateral crown height ${ }^{17}$ according to the following groups:

1) $>3 \mathrm{~mm}$

2) $\leq 3 \mathrm{~mm}>2 \mathrm{~mm}$

3) $\leq 2 \mathrm{~mm}>1 \mathrm{~mm}$

4) $\leq 1 \mathrm{~mm}$ and $0 \mathrm{~mm}$

4. The buccal and lingual margin design of tooth preparations (Shoulder, Chamfer, Feathered or No clear margin). This was examined using magnifier lens ${ }^{32}$.

5. Axial convergence angle between opposing walls of prepared dies $^{32}$. This was measured using Toolmaker microscope. The prepared die was held in a vertical position over a graded rotary table, and viewed under the microscope. The vertical line of the lens should overlap one of the axial walls. The graded rotary table that hold the die was then adjusted and turned around until the line overlaps the opposing axial wall. The angle formed between the two positions of the line represents the convergence angle The variables were assessed as if:

1) Less than $6^{\circ}$ axial convergence

2) Between $6^{\circ}$ and $10^{\circ}$ axial convergence

3) More than $10^{\circ}$ axial convergence

6 . Finish line depth was assessed using the Toolmaker Microscope. The die was held vertically over the graded table and viewed under the microscope. The vertical line of the microscope lens was adjusted vertically to across the internal line angle of the finish line. While keeping the line at the same position and direction, the table moves laterally until the line become tangent to the external surface of the prepared tooth; the distance which the table moves calculated as finish line depth ${ }^{32}$.

The depth of the finish line was assessed as if:

1) Less than $0.5 \mathrm{~mm}$ depth

2) Between 0.5 and $1.5 \mathrm{~mm}$ depth

3) More than $1.5 \mathrm{~mm}$

7. Depth continuity of the finish line was measured using the Toolmaker Microscope in the 4 aspects of the prepared die. This was assessed as follows:

1) Continuous finish line

2) Not continuous finish line

8. The labial planes of tooth preparation were assessed using the Toolmaker Microscope. The die was held horizontally over the graded table and viewed under the microscope. The M-D axis of the prepared tooth was held perpendicular to the graded table. The external $x-y$ axis of the microscopic lens was adjusted to across the labial surface of the die. If the $x$-axis kept in close contact from the cervical third to the incisal third of the prepared die this indicated non-anatomical (1 plane) labial tooth reduction and if the $\mathrm{x}$-axis kept in close contact till middle third only and then deviated from the labial surface, this indicated anatomical labial (2 planes).

Frequency tables were used to describe criteria of aspects of preparations examined on dies and numbers of preparations which followed the identified criteria. To investigate the inter-examiner reproducibility of the scoring systems, a random subsample of dies $(n=20)$ was selected and rescored after 7 days and the results compared ${ }^{41}$.

\section{RESULTS}

Of the total 100 casts examined in this study, 62 casts containing 141 dies (67\%) were preparations for IPS Empress, while 38 casts containing 67 dies were preparation for In-Ceram (33\% of the total dies examined). The total number of dies examined was 208.

A supragingival finish line was noticed in 12 dies $(6 \%)$, while $53 \%$ (110 dies) demonstrated equigingival margins, 36\% (76 dies) had subgingival margins and 5\% (10 dies) demonstrated no clear margin (Table 1).

It was possible to measure the total reduction in the buccolingual and mesiodistal planes and incisal reduction in 55 dies (39\%) of the 141 IPS Empress preparations, and in 39 of the 67 dies of In-Ceram samples (58\%). The remaining was unsuitable for the measurements due to lack of contralateral tooth. Of the total suitable 94 dies, $54 \%$ demonstrated overpreparation (>3 mm), 33\% exhibited the recommended depth of preparation $(<3>2)$ and $13 \%$ showed underpreparation. $(<2$ $\mathrm{mm}$ ) (Table 2).

Twenty percent (42 dies) of the 208 dies 
demonstrated a shoulder finish line while a chamfer margin design was noticed in $39 \%$. Twenty-nine percent and $12 \%$ of samples had either a feathered or no clear margin design, respectively (Table 3 ).

Table 4 shows that of the 94 dies, $18 \%$ demonstrated underpreparation incisally $(<1$ $\mathrm{mm}$ ). Only $17 \%$ of all RBC preparations were found to follow the recommended anatomical labial preparations.
Table 5 shows the degree of axial convergence angle between opposing walls of the RBC tooth preparations. Seventy one percent (148 dies) exceeded the recommended angle in a range between 21 and 28 degrees.

Forty three tooth preparations were found to have the recommended depth of the finish line and $30 \%$ were found to have under prepared depth of finish line (Table 6). Continuous finish line depth

Table 1- Tooth preparation margin positions in relation to the gingival margin position on the buccal/labial and lingual/palatal aspect

\begin{tabular}{lcccccc}
\hline Type of RBC & $\begin{array}{c}\mathbf{>} \mathbf{~ m m} \\
\text { supragingival }\end{array}$ & $\begin{array}{c}\mathbf{\leq 2} \mathbf{~ m m} \\
\text { supragingival }\end{array}$ & level & subgingival & $\begin{array}{c}\text { No clear } \\
\text { margin }\end{array}$ & Total \\
\hline IPS Impress & $3(2 \%)$ & $5(3.5 \%)$ & $70(50 \%)$ & $58(41 \%)$ & $5(3.5 \%)$ & $141(100 \%)$ \\
In-Ceram & $2(3 \%)$ & $2(3 \%)$ & $40(60 \%)$ & $18(27 \%)$ & $5(7 \%)$ & $67(100 \%)$ \\
Total & $5(2.5 \%)$ & $7(3.5 \%)$ & $110(53 \%)$ & $76(36 \%)$ & $10(5 \%)$ & $208(100 \%)$ \\
\hline
\end{tabular}

${ }^{*}$ Percentage $(\%)$ within each group. $\mathrm{RBC}=$ resin-bonded all-ceramic crowns

Table 2- Total amount of tooth tissue reduction in the buccolingual and mesiodistal planes of the RBC tooth preparations

\begin{tabular}{|c|c|c|c|c|c|}
\hline Type of RBC & $>3 \mathrm{~mm}$ & $\leq 2 \mathrm{~mm}>3 \mathrm{~mm}$ & $\leq 2 \mathrm{~mm}>1 \mathrm{~mm}$ & $\leq 1 \mathrm{~mm} \& 0 \mathrm{~mm}$ & Total \\
\hline IPS Impress & $33(60 \%)$ & $17(31 \%)$ & $3(5 \%)$ & $2(4 \%)$ & $55(100 \%)$ \\
\hline In-Ceram & $18(46 \%)$ & $14(36 \%)$ & $5(13 \%)$ & $2(5 \%)$ & $39(100 \%)$ \\
\hline Total & $51(54 \%)$ & $31(33 \%)$ & $8(9 \%)$ & $76(36 \%)$ & $94(100 \%)$ \\
\hline
\end{tabular}

$\mathrm{RBC}=$ resin-bonded all-ceramic crowns

Table 3- Buccal/labial and lingual/palatal margin designs for the resin-bonded all-ceramic crowns (RBC) tooth preparations

\begin{tabular}{lccccc}
\hline Type of RBC & Shoulder & Chamfer & Feathered & No clear margin & Total \\
\hline IPS Impress & $30(21 \%)$ & $61(43.5 \%)$ & $36(25.5 \%)$ & $14(10 \%)$ & $141(100 \%)$ \\
In-Ceram & $12(18 \%)$ & $19(28.5 \%)$ & $25(37 \%)$ & $11(16.5 \%)$ & $67(100 \%)$ \\
Total & $42(20 \%)$ & $80(39 \%)$ & $61(29 \%)$ & $25(12 \%)$ & $208(100 \%)$ \\
\hline
\end{tabular}

Table 4 - Amount of incisal reduction of the tooth preparation

\begin{tabular}{|c|c|c|c|c|c|}
\hline Type of RBC & $>3 \mathrm{~mm}$ & $\leq 3 \mathrm{~mm}>2 \mathrm{~mm}$ & $\leq 2 \mathrm{~mm}>1 \mathrm{~mm}$ & $\leq 1 \mathrm{~mm} \mathrm{\&} 0 \mathrm{~mm}$ & Total \\
\hline IPS Impress & $11(20 \%)$ & $15(27 \%)$ & $19(35 \%)$ & $10(18 \%)$ & $55(100 \%)$ \\
\hline In-Ceram & $5(13 \%)$ & $12(31 \%)$ & $15(38 \%)$ & $7(18 \%)$ & $39(100 \%)$ \\
\hline Total & $16(17 \%)$ & $27(29 \%)$ & $34(36 \%)$ & $17(18 \%)$ & $94(100 \%)$ \\
\hline
\end{tabular}

$\mathrm{RBC}=$ resin-bonded all-ceramic crowns

Table 5- Axial convergence angle between opposing walls of prepared die

\begin{tabular}{lcccc}
\hline Type of RBC & $<6^{\circ}$ & $\mathbf{6}^{\circ}-10^{\circ}$ & $>10^{\circ}$ & Total \\
\hline IPS Impress & $11(8 \%)$ & $25(18 \%)$ & $105(74 \%)$ & $141(100 \%)$ \\
In-Ceram & $5(8 \%)$ & $19(28 \%)$ & $43(64 \%)$ & $67(100 \%)$ \\
Total & $16(8 \%)$ & $44(21 \%)$ & $148(71 \%)$ & $208(100 \%)$ \\
\hline
\end{tabular}

$\mathrm{RBC}=$ resin-bonded all-ceramic crowns 
Table 6- Finish line depth

\begin{tabular}{lcccc}
\hline Type of RBC & $\mathbf{< . 5} \mathbf{~ m m}$ & $\mathbf{0 . 5}-\mathbf{1 . 5} \mathbf{~ m m}$ & $\mathbf{> 1 . 5} \mathbf{~ m m}$ & Total \\
\hline IPS Impress & $50(35 \%)$ & $55(39 \%)$ & $36(26 \%)$ & $141(100 \%)$ \\
In-Ceram & $12(18 \%)$ & $35(52 \%)$ & $20(30 \%)$ & $67(100 \%)$ \\
Total & $62(30 \%)$ & $90(43 \%)$ & $56(27 \%)$ & $208(100 \%)$ \\
\hline
\end{tabular}

$\mathrm{RBC}=$ resin-bonded all-ceramic crowns

was observed in $77 \%$ of preparations.

The results showed that ( $83 \%)$ of all RBC preparations had non-anatomical preparations, while only $(17 \%)$ had the recommended anatomical labial preparations (Table 6).

\section{Kappa statistics}

The Kappa statistics quantifying the interexaminer variability for the various measurements performed showed 130 variables out of 160 having the same scores between first and second measurements readings while 30 variables showed dissimilar agreements between first and second measurements readings. The Kappa value was $0.875^{34}$.

\section{DISCUSSION}

The use of RBC crowns has increased and there appears to be a wide variety of clinical indications, particularly in situations in which a minimal preparation is indicated or in which where there are already tooth substance loss ${ }^{13}$. Currently, crowns such as Inceram (Vivadent), IPS Empress (Ivoclar) and others, bonded with resin cement, can provide acceptable service when they are performed in the right way that should be.

The higher percentages of IPS Empress preparations compared with In Ceram as found in this study may be due to the fact that dental technicians tend to work with IPS Empress more than with In-Ceram crowns, as the fabrication for the IPS Empress is less time consuming and it gives the same aesthetic result while having the same cost of fabrication as pointed out verbally by dental technicians.

Ideally, finish line position should be placed supragingivally on sound tooth tissue, but in reality this is often not possible ${ }^{29}$. Sometimes aesthetics dictates a margin to be placed subgingivally and in these situations it should extend by $0.5-1 \mathrm{~mm}$, but certainly not more than half the depth of the gingival sulcus, to ensure the epithelial attachment is not compromised ${ }^{29}$. The placement of RBC margins subgingivally is critical because of the possibility of microleakage if the margins are placed either on dentin or cementum ${ }^{20,30}$. It has been shown that the bonding of luting material will be compromised if moisture control is inadequate, which is the case in subgingival preparations ${ }^{9}$. However, subgingival finish lines frequently are required in cases of inadequate occluso-cervical dimension needed for retention and resistance form, to extend beyond dental caries, fractures, or erosion/abrasion, to produce a cervical crown ferrule on endodontically treated teeth and to improve the aesthetics of discolored teeth and certain restorations. In such circumstances, it may be advisable to think in terms of a conventional crown ${ }^{20}$.

In the present study, $36 \%$ of the tooth preparations of RBCs had subgingival margin on the labial aspect which is not recommended for resin bonded crowns. Several studies have also shown that subgingival finish lines for RBCs were produced in general dental practice ${ }^{7,32}$.

Sufficient axial reduction is important to provide structural durability for the restoration and avoid over reduction. The use of depth orientation groove burs would be a useful method to ensure adequate axial tooth reduction. In addition, overpreparation of the teeth negates the advantages that RBCs demonstrate and may lead to loss of pulpal vitality and periradicular pathology ${ }^{11,25}$.

However, underpreparation will result in inappropriate labial and palatal contours, leading to compromised aesthetics. Overbulking of the RBCs at the gingival margin may be necessary to allow for adequate material strength, which results in a poor emergence profile ${ }^{26}$. Several studies considered an improper emergence profile as a significant etiologic factor in the marginal inflammation associated with crowns 22,23 .

Insufficient labial reduction, particularly near the finish line, may also result in distortion of the ceramic during fabrication and clinical service which leads to poor marginal adaptation, debonding, and long-term cement failure, all of which have been cited as major factors in the failure of ceramic crowns ${ }^{22}$.

In the present investigation, the method used to measure the total amount of tooth reduction merely took into account the total amount of tooth reduction in one plane and not the individual axial wall preparation depth, therefore one aspect of the tooth may have been appropriately prepared, whilst the other may have been incorrect. However, the measurements still served as a guide for axial wall tooth reduction. Ninety-four (45\%) samples were 
suitable for analysis of the total reduction in the buccolingual and mesiodistal planes, the remaining were not used due to lack of an unrestored contralateral or missing contralateral tooth.

As much as $54 \%$ of the RBCs showed overpreparation with a tendency to overprepare the teeth on the mesiodistal plane rather than buccolingual plane. This is in agreement with a previous study ${ }^{32}$. The results of this study also indicated that $12 \%$ of samples showed underpreparation of axial walls, which may result in a bulbous restoration with plaque retention leading to periodontal problems and/or an unsightly emergence profile. The marginal designs of ceramic crowns have been reviewed recently. Several designs have been advocated to optimize aesthetics, minimize marginal openings, and reduce stress concentration at the marginal aspect ${ }^{25}$. It has been reported that strong correlations exist between finish lines designs and all-ceramic crowns strength. Crowns with a chamfer finish line were significantly weaker than those with a shoulder finish line ${ }^{16}$.

The flat shoulder margin provides the required aesthetics and marginal stability necessary during porcelain firing, and it is the most suitable for a labial finishing line for anterior all-ceramic crowns ${ }^{26,27,31}$. However, when resin cement was used with internally etched all-ceramic crowns, there was no significant strength reduction in a laboratory study or in a longitudinal retrospective clinical evaluation of all-ceramic crowns compared with non etched all-ceramic crowns ${ }^{6}$. Therefore, a shoulder or definitive chamfer finish lines are recommended for all-ceramic crowns that are not etched and bonded to the teeth.

Beveled or feather margins can lead to higher chance for ceramic fracturing during the seating or at some point after cementation. The technician, in an attempt to strengthen the margin, may overbuild the RBC, which may result in a bulbous margin with plaque retention leading to periodontal problems and/or an unsightly emergence profile ${ }^{6}$.

With regard to the marginal design, 59\% of the samples demonstrated a shoulder or chamfer margin design on the buccal/labial and lingual/ palatal aspects, $29 \%$ and $12 \%$ had either a feathered or no clear margin design respectively. In a similar study $84 \%$ of the buccal and $79 \%$ of the lingual margins had shoulder or chamfer preparations while $16 \%$ on the buccal and $21 \%$ on the lingual aspects demonstrated a feathered margin design or no detectable margin ${ }^{32}$.

Proper incisal reduction is of importance as it will improve subsequent preparation access and helps to ensure correct proportioning of axial reduction planes. It was proposed that incisal/ occlusal surfaces reduction should be $2 \mathrm{~mm}$ because this depth permits the development of normal morphology and has been identified as safe and reasonable amount to remove from tooth ${ }^{15}$. It is also important to provide an adequate bulk of porcelain in areas exposed to heavy loading. The number of dies that was suitable for measurements of incisal reduction were 55 Empress and 39 InCeram, the other dies were unsuitable for the test due to absent of the contralateral tooth or the present of a restoration on the contralateral tooth. The results showed that $18 \%$ of Empress and $18 \%$ of the In-Ceram tooth preparation dies demonstrated under preparation occlusally. Sutton and McCord $^{32}$ (2001) found that tooth preparations for low fusing, Chameleon Fortress and Empress RBCs demonstrated under preparation occlusally ${ }^{32}$.

Anatomical tooth preparation is important to achieve good aesthetics and preserve dental tissues. Long clinical crowns will often need to undergo three plane reductions. Poor contour of the restoration may in addition result in an unaesthetic restoration since the eye perception of tooth form is of a higher order than tooth shade ${ }^{23}$.

Non-anatomical preparations may also result in a preparation with an overcontoured or "bulky" restoration and may also result in periodontal problems unless oral hygiene standards are exceptionally high ${ }^{23}$.

Regarding the buccal/labial planes of the RBC tooth preparations, $83 \%$ of all RBC preparations had non-anatomical preparations while only $17 \%$ had the recommended anatomical labial preparations. In a similar study they, found that the majority of teeth (56\%) were found to be prepared with respect to tooth morphology, while $42 \%$ were prepared with only one plane of preparation on the buccal/ labial aspect ${ }^{32}$.

It is helpful to know that many tapered burs have a $5-6^{\circ}$ convergence angle which can be used to survey preparation taper by holding the hand piece in the same plane for all axial surfaces ${ }^{6}$. Resin bonded crowns are the important exception to the rule of minimizing taper, especially RBCs which may benefit from having tapers of about $20^{\circ}$ to avoid generating high seating hydrostatic pressures during luting resulting in crown fracture ${ }^{6}$. Therefore, it is proposed that total occlusal convergence ideally should range between 10 and 20 degrees $^{27}$.

The results of axial convergence angle between opposing walls for RBCs showed $29 \%$ of the RBC tooth preparations having the recommended axial convergence angle ( $5^{\circ}$ to $10^{\circ}$ ) and $71 \%$ have exceeded the recommended angle in a range between 21 and 28 degrees.

As a general rule when using porcelain crowns, adequate clearance is required to achieve good aesthetics. This is achieved with a shoulder or heavy chamfer of $0.8-1 \mathrm{~mm}$ width for RBCs. However, 
shoulders of these depths may compromise tooth strength and pulp health especially for small teeth such as mandibular incisors. A similar problem occurs on teeth with long clinical crowns because of the narrowing of their diameter in the cervical region. Therefore, the recommended finish line depths for all-ceramic crowns have ranged from 0.5 to $1.0 \mathrm{~mm}^{10,24,27,28}$.

Regarding the finish line depth of RBC preparations, the majority of RBC tooth preparations $(43 \%)$ had the recommended depth $(0.5 \mathrm{~mm}$ to 1.5 $\mathrm{mm}$ ) of the finish line. This result shows that GDPs in Jordan are aware of the proper finish line depth required for all-ceramic crowns. Thirty percent had underpreparation depth of finish line, which may be related to the finding that $29 \%$ of the GPDs in the present investigation had feathered margin design of the finish line. Twenty-seven percent had overpreparation finish line depth, which, as mentioned above, will lead to poor aesthetic outcomes and may compromise tooth strength and pulp health.

Incomplete and/or non-uniform shoulder causes the porcelain in the cervical areas to vary significantly in thickness, with a potential for premature fracture during fabrication, in the process of seating, or after cementation ${ }^{1}$. Seventy-seven percent of RBC tooth preparations had continues finish line depth, while $23 \%$ had non-continuous finish line depth.

Finally, from the results obtained in this study, it has been shown that there are wide variations in the preparations of RBC crowns for anterior teeth in general dental practice in Jordan. The results also showed the most common clinical errors in preparation of anterior teeth for all-ceramic crowns among GDPs in Jordan and focused on the most accepted recommendation needed when preparing anterior teeth for all-ceramic crowns, which will lead to increased life time expectancy of the prosthesis, enhanced clinical performance, and increased procedural efficiency. Long-term studies in general dental practice, where the majority of the RBC restorations are placed, are still needed. Graduate education for GDPs who were not trained to use RBCs as undergraduates is probably necessary to improve the knowledge of the required preparation designs.

\section{CONCLUSIONS}

This study showed that preparations for RBCs of the Jordanian clinicians' work investigated varied widely. Therefore, under the tested conditions, the following conclusions may be drawn:

GDPs followed the guidelines for RBC preparation finish lines by preparing either shoulder or chamfer finish line. However, there were a number of cases with either no detectable margin or feathered margins. They were found to locate the finish line margins preparation subgingivally with no clear margins in some cases.

The majority of cases had overpreparation not taking in consideration tooth morphology, which made most preparation done in one plane of reduction.

The majority of cases had the recommended incisal reduction.

The majority of dies examined exceeded the recommend axial convergence angle in a range of 21 and 28 degrees.

The majority of dies examined had the recommend finish line depth and showed continuity of the finish line depth.

Relevant guidelines for the preparations of RBCs are not being entirely adhered to in private practice in Jordan.

\section{REFERENCES}

1- Adams DC. The ten most common all-ceramic preparation errors: a doctor/technician liaison's perspective. Dent Today. 2004;23:94, 96-9.

2- Anusavice KJ. Phillips' science of dental materials. $11^{\text {th }}$ ed. St Louis: Elsevier; 2003. p. 431-8, 539, 702.

3- Begazo CC, Van Der Zel JM, Van Waas MA, Feilzer AJ. Effectiveness of preparation guidelines for an all-ceramic restorative system. Am J Dent. 2004;17(6):437-42.

4- Bernal G, Jones RM, Brown DT, Munoz CA, Goodacre CJ. The effect of finish line form and luting agent on the breaking strength of Dicor crowns. Int J Prosthodont. 1993;6:286-90.

5- Bertolotti RL. Removal of "black-line" margins and improving esthetics of porcelain-fused- to-metal crowns: update in technique. Quintessence Int. 1990;21:643-6.

6- Blair FM, Wassell RW, Steele JG. Crowns and other extracoronal restorations: preparations for full veneer crowns. $\mathrm{Br}$ Dent J. $2002 ; 192: 561-71$.

7- Brunton PA, Wilson NH. Preparations for porcelain laminate veneers in general dental practice. Br Dent J. 1998;184:553-6.

8- Burke FJ, Fleming GJ, Nathanson D, Marquis PM. Are adhesive technologies needed to support ceramics? An assessment of the current evidence. J Adhes Dent. 2002;4:7-22.

9- Burke FJ, Watts DC. Fracture resistance of teeth restored with dentin-bonded crowns. Quintessence Int. 1994;25:335-40.

10- Chiche GJ, Pinault A. Aesthetics of anterior fixed prosthodontics. Chicago: Quintessence Publishing Co; 1994. p. 86, 91, 102-3.

11- Christensen GJ. Tooth preparation and pulp degeneration. J Am Dent Assoc. 1997;128:353-4.

12- Clyde JS, Gilmour A. Porcelain veneers: a preliminary review. Br Dent J.1988;164:9-14.

13- Crothers AJR, Wassell RW, Allen R. The resin-bonded porcelain crown: a rationale for use on anterior teeth. Dent Update. 1993;20:388-95.

14- De Jager N, Pallav $P$, Feilzer AJ. The influence of design parameters on FEA-determined stress distribution in CAD-CAM produced all ceramic crowns. Dent Mater. 2005;21:242-51.

15- Douglas RD, Przybylska M. Predicting porcelain thickness required for dental shade matches. J Prosthet Dent. 1999;82:1439.

16- Doyle MG, Goodacre CJ, Munoz CA, Andres CJ. The effect of tooth preparation design on the breaking strength of Dicor crowns: 3. Int J Prosthodont. 1990;3:327-40.

17- Etemadi S, Smales RJ, Drummond PW, Goodhart JR. Assessment of tooth preparation designs for posterior resinbonded porcelain restorations. J Oral Rehabil. 1999;26:691-7. 
18- Hilgert E, Buso L, Neisser MP, Bottino MA. Evaluation of marginal adaptation of ceramic crowns depending on the marginal design and the addition of ceramic. Brazil J Oral Scien. 2004;3(11):619-23.

19- Karlsson S, Landahl I, Stegersjö G, Milleding P. A clinical evaluations of ceramic laminate veneers. Int J Prosthodont. 1992;5:447-51.

20- Lacy AM, Wada C, Du W, Watanabe L. In vitro microleakage at the gingival margin of porcelain and resin veneers. J Prosthet Dent. 1992;67:7-10.

21- Mayers RE. Standards of human occlusal development. Ann Arbor: Center for Human Growth and Development; 1976. p. 45-8. 22- Reeves WG. Restorative margin placement and periodontal health. J Prosthet Dent. 1991;66:733-6.

23- Rimmer SE, Mellor AC. Patients' perceptions of aesthetics and technical qualities in crowns and fixed partial dentures. Quintessence Int. 1996;27:155-62.

24- Rosenstiel SF, Land MF, Fujimoto J. Contemporary fixed prosthodontics. $2^{\text {nd }}$ ed. St. Louis: Mosby-Year Book; 1995. p. 137-8, 170-3, 184-5, 229.

25- Saunders WP, Saunders EM. Prevalence of periradicular periodontitis associated with crowned teeth in an adult Scottish subpopulation. Br Dent J. 1998;185:137-40.

26- Seymour KG, Samarawickrama DY, Lynch EJ. Metal ceramic crowns - a review of tooth preparation. Eur J Prosthodont Restor Dent. $1999 ; 7: 79-84$.
27- Shillingburg HT, Hobo S, Whitsett LD, Jacobi R, Brackett SE. Fundamentals of fixed prosthodontics. $3^{\text {rd }}$ ed. Chicago: Quintessence Publishing Co; 1997. p. 120, 139-42, 151-52.

28- Shillingburg HT, Jacobi R, Brackett SE: Fundamentals of tooth preparations: for cast metal and porcelain restorations. Chicago: Quintessence Publishing Co.; 1987. p. 13.

29- Silness J. Periodontal conditions in patients treated with dental bridges. 3 . The relationship between the location of the crown margin and the periodontal condition. J Periodontal Res. 1970;5:225-9.

30- Sim C, Neo J, Chua EK, Tan BY. The effect of dentine bonding agents on the microleakage of porcelain veneers. Dent. Mater. $1994 ; 10: 278-81$.

31- Smith BGN. Planning and making crown and bridges. $3^{\text {rd }}$ ed. Saint Louis: Mosby; 1998. p. 41-6.

32- Sutton AF, McCord JF. Variations in tooth preparations for resin-bonded all-ceramic crowns in general dental practice. $\mathrm{Br}$ Dent J. 2001;191(12):677-81.

33- Thompson VP, Rekow DE. Dental ceramics and the molar crown testing ground. J Appl Oral Sci. 2004;12(Sp. Iss.):26-36. 34- Viera AJ, Garrett JM. Understanding interobserver agreement: the kappa statistic. Fam Med. 2005;37:360-3. 